\title{
Epithelial myosin light chain kinase expression and activity are upregulated in inflammatory bowel disease
}

\author{
Stephanie A Blair ${ }^{1}$, Sunanda V Kane ${ }^{2}$, Daniel R Clayburgh ${ }^{1}$ and Jerrold R Turner ${ }^{1}$ \\ ${ }^{1}$ Department of Pathology, The University of Chicago, Chicago, IL, USA and ${ }^{2}$ Department of Medicine, \\ The University of Chicago, Chicago, IL, USA
}

\begin{abstract}
The intestinal epithelial barrier is frequently disrupted in inflammatory bowel disease (IBD) and this has been proposed to play a role in disease pathogenesis and reactivation. In vitro studies show that cytokine-induced epithelial barrier dysfunction can be mediated by increased myosin light chain kinase (MLCK) expression and subsequent myosin II regulatory light chain (MLC) phosphorylation. However, this has never been examined in human disease. The aim of these studies, therefore, was to determine whether MLCK is upregulated in the intestinal epithelium of IBD patients. MLCK expression and MLC phosphorylation in human intestinal resection and biopsy specimens were determined by quantitative immunofluorescence microscopy and correlated with clinical and histopathological data. The data show that ileal epithelial MLCK expression was mildly upregulated in inactive IBD. Expression increased further in active disease, with progressive increases in MLCK expression correlating positively with histological disease activity. This correlation between activity and MLCK expression was also seen in individual patients where areas of differing disease activity were analyzed. Colonic epithelial MLCK expression was similarly increased in active IBD and these increases also correlated positively with disease activity, both in individual patients and the overall study group. To evaluate MLCK enzymatic activity, MLC phosphorylation was assessed in snap-frozen colon biopsies. MLC phosphorylation was significantly increased in biopsies with active, but not inactive, IBD. Therefore, these data show that MLCK expression and enzymatic activity are increased in IBD. Moreover, the correlation with disease activity suggests that MLCK upregulation may contribute to barrier dysfunction and IBD pathogenesis.
\end{abstract}

Laboratory Investigation (2006) 86, 191-201. doi:10.1038/labinvest.3700373; published online 9 January 2006

Keywords: myosin; Crohn's disease; ulcerative colitis; tight junctions; tumor necrosis factor

A primary function of intestinal epithelia is the maintenance of a barrier to prevent unregulated mixing of luminal and interstitial compartments. While the relevance of epithelial barrier dysfunction to disease pathogenesis remains a subject of active investigation, it is well accepted that compromised barrier function is a common feature of infectious, ischemic, and immune-mediated intestinal disease. ${ }^{1}$ This has been well defined in Crohn's disease (CD), where barrier defects can be detected in patients as well as a subset of their healthy relatives. ${ }^{2-4}$ Moreover, barrier dysfunction may precede clinical evidence of disease ${ }^{5}$ and may serve as a marker of impending disease reactivation. ${ }^{6}$ Barrier dysfunc-

Correspondence: Dr JR Turner, MD, PhD, Department of Pathology, The University of Chicago, 5841 South Maryland Avenue, MC 1089, Chicago, IL 60637, USA.

E-mail: jturner@bsd.uchicago.edu

Received 12 October 2005; revised 1 November 2005; accepted 2 November 2005; published online 9 January 2006 tion in CD patients has also been positively correlated with systemic immune activation. ${ }^{7}$ This may, in part, be due to release of proinflammatory cytokines, including tumor necrosis factor (TNF). For example, barrier dysfunction in CD can be corrected by treatment with TNF-neutralizing antibodies. ${ }^{8}$ However, TNF-neutralizing antibodies exert a major part of their therapeutic effect by general suppression of TNF signaling between immune cells. Thus, in the absence of data explaining mechanisms of epithelial barrier dysfunction in CD and ulcerative colitis (UC), it is not possible to fully evaluate the significance of barrier dysfunction to the pathobiology of these diseases.

One clue to the mechanism of barrier dysfunction in inflammatory bowel disease (IBD) may come from in vitro studies showing that $\mathrm{TNF} \alpha$ induces barrier dysfunction in cultured intestinal epithelial monolayers. ${ }^{9,10}$ We have shown in vitro that TNFdependent barrier dysfunction requires myosin II activation by myosin light chain kinase (MLCK)- 
dependent myosin II regulatory light chain (MLC) phosphorylation. ${ }^{11}$ Consistent with this, MLCK inhibition corrects TNF-induced intestinal barrier dysfunction in vitro ${ }^{11}$ as well as in vivo intestinal barrier dysfunction in a mouse model of TNFdependent diarrhea. ${ }^{12}$ Such MLCK-dependent regulation of epithelial barrier function has been described in response to other physiological and pathophysiological stimuli and appears to be due to increased paracellular permeability resulting from tight junction regulation. ${ }^{13-15}$ In addition, we and others have recently shown that in vitro TNFinduced barrier dysfunction in model intestinal epithelia requires increased MLCK expression. ${ }^{16,17}$ However, in vivo MLCK expression has not been examined in humans or experimental animals.

The aim of these studies was to determine whether MLCK expression and enzymatic activity are increased in the intestinal epithelium of IBD patients. We analyzed surgical resection and biopsy specimens from a total of 43 IBD patients and control subjects. The data show that both MLCK expression and activity are increased in IBD and that these increases correlate with disease activity. These results support a role for MLCK in inducing the intestinal barrier dysfunction seen in IBD patients.

\section{Materials and methods}

\section{Sample Collection}

Snap-frozen resection and biopsy specimens were obtained from patients followed at The University of Chicago Hospitals. Resection specimens were sampled at multiple sites, snap frozen as fullthickness sections in Optimal Cutting Temperature media within $60 \mathrm{~min}$ of surgical resection, and stored at $-80^{\circ} \mathrm{C}$. Biopsy specimens were obtained from patients undergoing colonoscopy for IBD. Control samples were obtained from patients undergoing colonoscopy as part of a routine program of polyp surveillance. Biopsy specimens were snap frozen in Optimal Cutting Temperature within $1 \mathrm{~min}$ of endoscopic resection and stored at $-80^{\circ} \mathrm{C}$. Clinical data for IBD patients were obtained by medical record review. All procedures were approved by The University of Chicago Institutional Review Board.

\section{Tissue Staining}

Frozen sections $(4 \mu \mathrm{m})$ were fixed in $1 \%$ paraformaldehyde (prepared in PBS) for 2 min, washed three times for 5 min with PBS, permeabilized using 0.5\% Igepal CA-630 (Sigma, St Louis, MO, USA) in PBS for $5 \mathrm{~min}$, and washed two more times in PBS. Sections were then blocked by incubation in PBS with $5 \%$ normal goat serum for $15 \mathrm{~min}$ prior to a 90 min incubation in primary antisera. After five washes in PBS with 1\% normal goat serum sections were incubated with secondary antisera for $60 \mathrm{~min}$. Fluorescent conjugates of phalloidin, to label Factin, and Hoeschst 33324, to label nuclei, were included with secondary antisera.

Primary antisera and concentrations used were: monoclonal mouse anti-pan MLCK, clone K36 (Sigma), $7.4 \mu \mathrm{g} / \mathrm{ml}$; affinity-purified anti-MLCK1specific rabbit antisera, ${ }^{18} 4 \mu \mathrm{g} / \mathrm{ml}$; affinity-purified rabbit anti-serine-19-phosphorylated myosin light chain antisera, ${ }^{19}$ and chicken antitotal myosin light chain antisera, ${ }^{12} 5 \mu \mathrm{g} / \mathrm{ml}$. Secondary antisera were all used at $8 \mu \mathrm{g} / \mathrm{ml}$ and were Alexa 488- or Alexa 594conjugated goat anti-mouse immunoglobulin (Invitrogen, Carlsbad, CA, USA), Alexa 488-conjugated goat anti-chicken immunoglobulin (Invitrogen), and Alexa 594-conjugated goat anti-rabbit immunoglobulin (Invitrogen). Alexa 488- and Alexa 350-conjugated phalloidin (Invitrogen) were used at $5 \mathrm{U} / \mathrm{ml}$ and Hoeschst 33324 (Invitrogen) was used at $1 \mu \mathrm{g} / \mathrm{ml}$.

\section{Immunofluorescence Microscopy and Image Analysis}

Sections were imaged using a DM4000 epifluorescence microscope (Leica Microsystems Inc., Bannockburn, IL, USA) equipped with 41004, 41017, and 31000v2 zero-pixel shift filter cubes (Chroma Technology, Rockingham, VT, USA) and a 12-bit Coolsnap HQ camera (Roper Scientific, Tucson, AZ, USA) controlled by MetaMorph 6.2 (Universal Imaging Corporation, Downingtown, PA, USA).

Images were analyzed using MetaMorph 6.2. For analysis of MLCK and MLCK1 expression tissues were double labeled for the kinase and F-actin. The region of the apical actomyosin ring was identified using the F-actin image. Intensities for the pixels corresponding to this region in the MLCK or MLCK1 images were then exported to Microsoft Excel (Microsoft Corporation, Redmond, WA, USA). Data were limited to discard outlying pixel intensities, as previously described. ${ }^{19}$ Mean pixel intensity for each sample was then normalized to a similarly analyzed standard control tissue (ileum or colon, corresponding to the diseased tissue used) that was stained daily to control for day-to-day staining variation.

Analysis of MLC phosphorylation was performed on biopsies double labeled for phosphorylated MLC and total MLC. To avoid potential artifact due to small variations in interval to freezing and day-today staining variation, MLC phosphorylation in each biopsy specimen was compared to a standard non-IBD control that was stained with each batch as well as colon biopsies from five control subjects without IBD. Preliminary studies showed that the intensity of total MLC labeling within these specimens was uniform except that in specimens with erosions or ulcerations MLC phosphorylation was difficult to assess reliably. This was partly due to the absence of epithelial cells from large areas of ulcerated biopsies, leaving insufficient epithelia 
available for analysis. These biopsies were therefore excluded from the study. In light of the lability of MLC phosphorylation, we limited our analysis to categorizing biopsies as having MLC phosphorylation less than, similar to, or greater than the control. With the exception of several ulcerated biopsies, which were excluded, no biopsies had MLC phosphorylation less than the control. In all cases noninflamed biopsies from subjects without IBD stained for phosphorylated MLC with intensities similar to controls.

\section{Hematoxylin and Eosin Staining and Activity Scoring}

At the time frozen sections were prepared for immmunofluorescent staining adjacent sections were collected and stained with hemotoxylin and eosin. These were reviewed by a gastrointestinal surgical pathologist (JRT) blinded to the clinical data to confirm that diseased tissues were involved by IBD and that normal controls were indeed normal. Samples were then graded for activity using on a four-tier scale; inactive, mild, moderate, severe (Figure 1). Tissue with inactive disease showed evidence of involvement by chronic IBD, including crypt architectural distortion, basal lymphoplasmacytic infiltrates, fibrosis, or epithelial metaplasia, but no active inflammatory infiltrates (neutrophils or eosinophils). Tissue with mild activity showed features of chronic IBD with rare intraepithelial neutrophils or eosinophils and increased numbers of neutrophils or eosinophils within the lamina propria. Moderately active disease was characterized by features of chronic IBD with frequent intraepithelial neutrophils or eosinophils, lamina propria distortion, and markedly increased numbers of neutrophils or eosinophils within the lamina propria. Tissue with severe activity showed features of chronic IBD with numerous crypt abscesses, erosions, or ulcerations and focal sheets of neutrophils or eosinophils within the lamina propria.

\section{Statistical Analysis}

Statistical analysis was performed with Stata 8.2. software (StataCorp, College Station, TX, USA). Normalized averages were grouped by disease activity grade (determined on hematoxylin- and eosin-stained slides) and compared to inactive disease samples or control samples using the Student's $t$-test. Fisher's exact test was used for proportions with calculation of Pearson's correlation coefficients. A $P$-value of $\leq 0.05$ was considered statistically significant by convention.

\section{Results}

\section{Patient Population}

The study group for analysis of MLCK expression consisted of 26 patients from The University of
Chicago Hospitals who underwent enteric resections that included right colon and ileum. A total of 20 patients underwent these procedures for IBD; five with UC and 15 with CD. Six patients were resected for right-sided adenocarcinoma. In these specimens, which served as controls, normal ileal and colonic margins of resection were analyzed. Additional demographic features of the patients whose resection specimens were studied are provided in Table 1.

For analysis of small intestinal disease activity and MLCK isoform expression, 26 separate fullthickness ileal sections from the $10 \mathrm{CD}$ patients were analyzed and compared to four control ileal sections; one each from four adenocarcinoma patients. Analysis of colonic disease activity and MLCK isoform expression was performed in 21 sections from IBD patients, 13 UC and eight CD, and five sections from adenocarcinoma patients.

Preliminary analyses showed that resection specimens were unsuitable for analysis of MLC phosphorylation, as detailed below. To avoid this, MLC phosphorylation was assessed in colonic mucosal biopsies snap frozen within $1 \mathrm{~min}$ of endoscopic resection. In all, 17 biopsies from 12 IBD patients, seven UC and five CD, and seven biopsies from five control subjects (without history of gastrointestinal disease who underwent colonoscopy for polyp surveillance) were analyzed.

For all specimens analyzed, histological activity was evaluated in serial sections stained by hematoxylin and eosin (Figure 1) that were immediately adjacent to sections used for immunofluorescent staining. Histological activity assessment and quantitative immunofluorescent analyses were performed independently and the data sets were not merged until all analyses were complete.

\section{Ileal Epithelial MLCK Expression is Increased in CD}

MLCK expression was assessed in ileal epithelium using quantitative immunofluorescence microscopy. In control tissues the subcellular localization of MLCK expression was similar to that previously reported in human jejunal enterocytes ${ }^{18}$ and was concentrated within the apical actomyosin ring and a less intense juxtanuclear pool (Figure 2). This distribution was qualitatively indistinguishable from that seen in ileal specimens with inactive CD. However, quantitative analysis of epithelial MLCK expression showed that it was increased in inactive CD. Normalized ileal enterocyte MLCK expression in CD specimens with inactive disease was $1.18 \pm 0.05 \quad(n=7)$, which was significantly $(P=0.03)$ greater than ileal enterocyte MLCK expression in control specimens.

Although observers were blinded to clinical data, it was immediately apparent that MLCK expression was increased in epithelia of tissues with active disease (Figure 2). The greatest variation in MLCK 
Table 1 Clinical features of patients whose resection specimens were studied

\begin{tabular}{|c|c|c|c|c|}
\hline & Ulcerative colitis & Crohn's disease & Total IBD & Control \\
\hline Number of patients & 5 & 15 & 20 & 6 \\
\hline Age mean (range) (years) & $46(25-51)$ & $37(21-69)$ & $39(21-69)$ & $70(60-79)$ \\
\hline Female:male & $0: 5$ & $11: 4$ & $11: 9$ & $2: 4$ \\
\hline Disease Duration & $7.4(1-14)$ & $9.3(2-19)$ & $8.85(1-19)$ & \\
\hline Family history & 0 & 2 & 2 & \\
\hline Previous surgeries & 0 & 7 & 7 & \\
\hline \multicolumn{5}{|l|}{ Smoking history \% (n) } \\
\hline Smoker & $0 \%(0)$ & $20 \%(3)$ & $15 \%(3)$ & \\
\hline Exsmoker & $40 \%(2)$ & $13 \%(2)$ & $20 \%(4)$ & \\
\hline Nonsmoker & $60 \%(3)$ & $67 \%(10)$ & $65 \%(13)$ & \\
\hline \multicolumn{5}{|l|}{ Current therapy } \\
\hline Cyclosporine & 5 & 9 & 14 & \\
\hline 6-Mercaptopurine & 0 & 4 & 4 & \\
\hline Infliximab & 0 & 1 & 1 & \\
\hline Antibiotics & 0 & 1 & 1 & \\
\hline None & 0 & 1 & 1 & \\
\hline \multicolumn{5}{|l|}{ Sites sampled \% (n) } \\
\hline Ileum & & $75.6 \%(28)$ & $56.0 \%(28)$ & $66.7 \%(4)$ \\
\hline Right colon & $84.6 \%(11)$ & $16.2 \%(6)$ & $34.0 \%(17)$ & $83.3 \%(5)$ \\
\hline Left colon & $15.4 \%(2)$ & $8.2 \%(3)$ & $10.0 \%(5)$ & \\
\hline Total & 13 & 37 & 50 & 6 \\
\hline \multicolumn{5}{|l|}{ Severity of active sites } \\
\hline Inactive & $15.4 \%(2)$ & $21.6 \%(8)$ & $20.0 \%(10)$ & \\
\hline Mild & $30.8 \%(4)$ & $37.8 \%(14)$ & $36.0 \%(18)$ & \\
\hline Moderate & $23.0 \%(3)$ & $27.1 \%(10)$ & $26.0 \%$ & \\
\hline Severe & $30.8 \%(4)$ & $13.5 \%(5)$ & $18.0 \%(9)$ & \\
\hline
\end{tabular}

expression was seen in cases with mild disease activity; six of nine cases had small increases similar to those associated with inactive disease while the remaining three cases had larger increases in MLCK expression (Figures 2 and 3). Overall, normalized ileal epithelial MLCK expression in specimens with mild activity was $1.54 \pm 0.21 \quad(n=9)$. Epithelial MLCK expression in ileal specimens with moderate disease activity was $1.59 \pm 0.18(n=7)$, a value similar to that in specimens with mild activity; normalized MLCK expression exceeded 1.5 in four of seven specimens (Figure 3). These 16 specimens were thus grouped for statistical analyses. In the group of ileal resection specimens with mild and moderate disease activity overall normalized MLCK expression was $1.56 \pm 0.14(n=16)$. This was significantly greater than expression in control specimens $(P=0.03)$ as well as in CD cases with inactive disease $(P=0.04)$.

Three ileal resection specimens with severe activity were available for analysis. MLCK expression was increased in each of these (Figures 2 and 3). Overall normalized MLCK expression averaged $2.16 \pm 0.43$, and expression exceeded 2.5 in two of three specimens $(P<0.01$ vs inactive CD). In these cases MLCK was still concentrated within the apical actomyosin ring, but the staining was far more intense and was readily seen throughout the cytoplasm such that exposures matched to those used for analysis of the other specimens resulted in oversaturation of the image (Figure 2). Owing to this oversaturation subcellular localization is best evaluated in the inset in which exposure was reduced by $50 \%$ (Figure 2). Overall, these data demonstrate that total ileal epithelial MLCK expression is increased in both inactive and active CD. Moreover, when the entire CD patient group was considered MLCK expression correlated positively with disease activity $(r=0.93)$.

We and others ${ }^{16,17}$ have recently shown that increased MLCK expression induced by TNF $\alpha$ in cultured cell lines is due to transcriptional upregulation of MLCK. ${ }^{20}$ Given the observed increase in MLCK expression in active CD, we sought to assess MLCK mRNA content using quantitative real-time RT-PCR. Unfortunately, these data were inconclusive with a large range of values obtained. While we do not know the reason for this technical difficulty and mRNA is reported to be stable in surgical specimens under the conditions used here, ${ }^{21}$ it is possible that the inflammatory responses present in tissues with active disease interfered with mRNA stability or other technical aspects of mRNA detection. We also attempted quantitative real-time RTPCR using colon biopsies from UC and CD patients. Again these were unsuccessful, likely a result of the low copy number of MLCK in intestinal epithelia and the small number of epithelial cells present in 

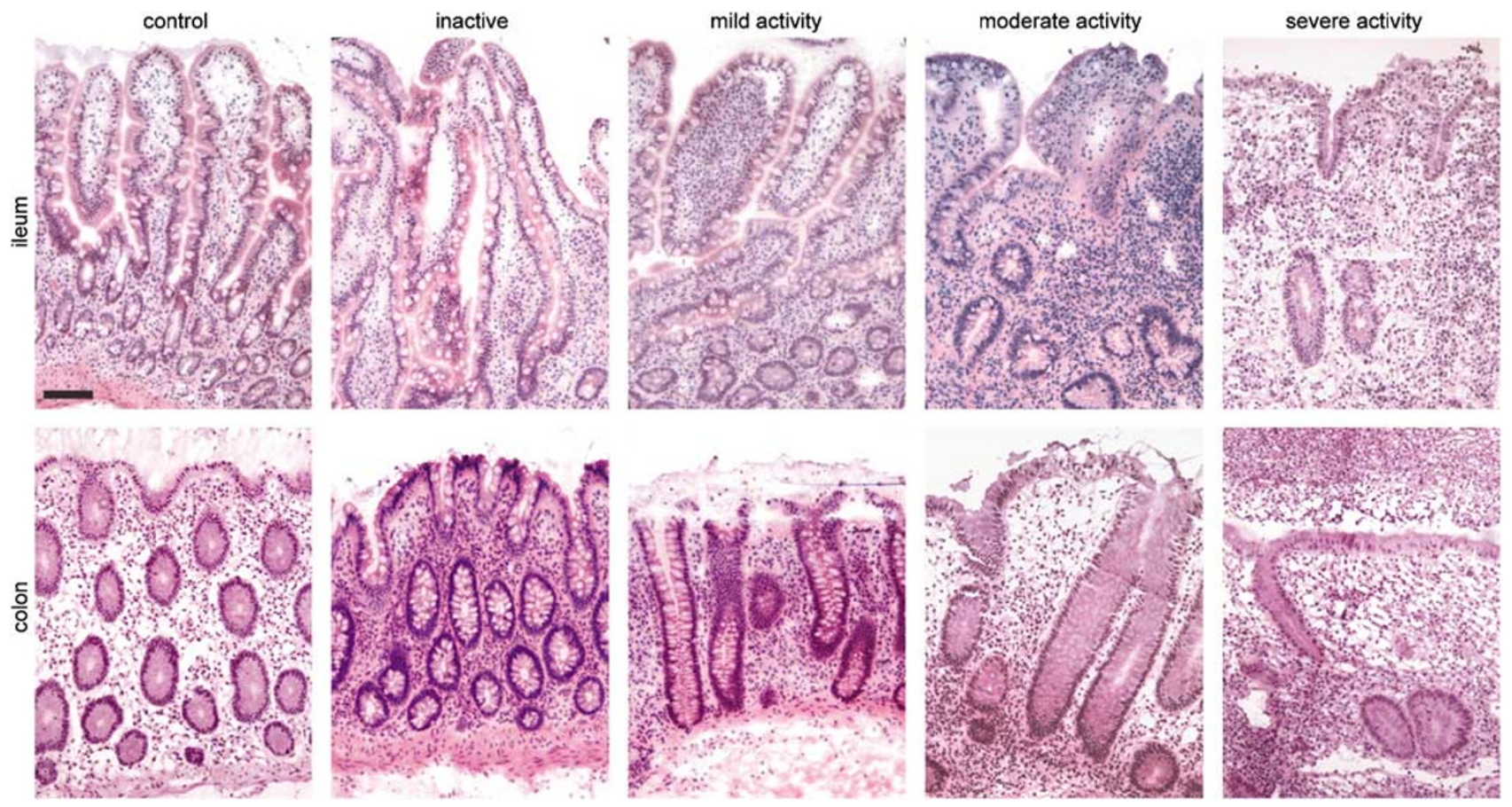

Figure 1 Histological grading of disease activity. Biopsy and resection specimens were graded for histologically evident disease activity using frozen sections adjacent to those used for quantitative immunofluorescence analysis of protein expression and phosphorylation. Details of the grading criteria are provided in the Materials and methods. Examples of ileal and colonic mucosa in each category are given. $\mathrm{Bar}=100 \mu \mathrm{m}$.
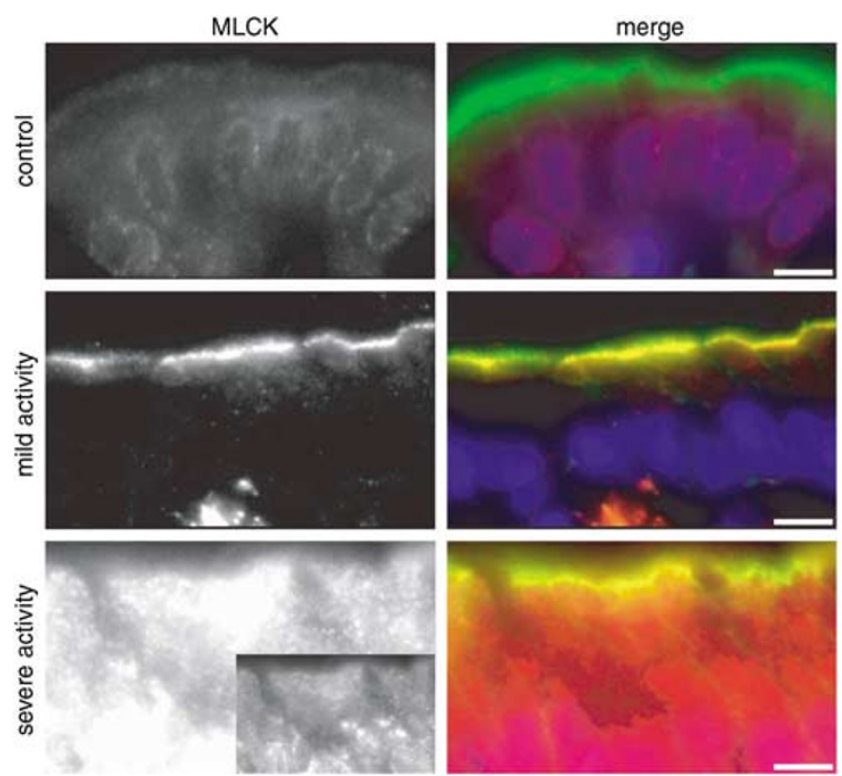

Figure 2 Ileal epithelial MLCK expression is increased in CD. In control tissue from a non-CD patient MLCK (red in the merged image) expression was limited, although detectable, in the cytoplasm and at the apical actomyosin ring of villus enterocytes. The merged image shows F-actin (green) and nuclei (blue) for orientation. In CD tissue with mild activity MLCK expression was increased and concentrated at the apical actomyosin ring (exposures are matched throughout). In tissue with severe activity MLCK expression was markedly increased. This resulted in saturation of the image when exposures were identical to those used for tissue without disease or with mild activity. The inset shows a $50 \%$ reduction of exposure for analysis of MLCK localization in tissue with severe disease. MLCK is detected at the apical actomyosin ring and throughout the cytoplasm. Bar $=5 \mu \mathrm{m}$.

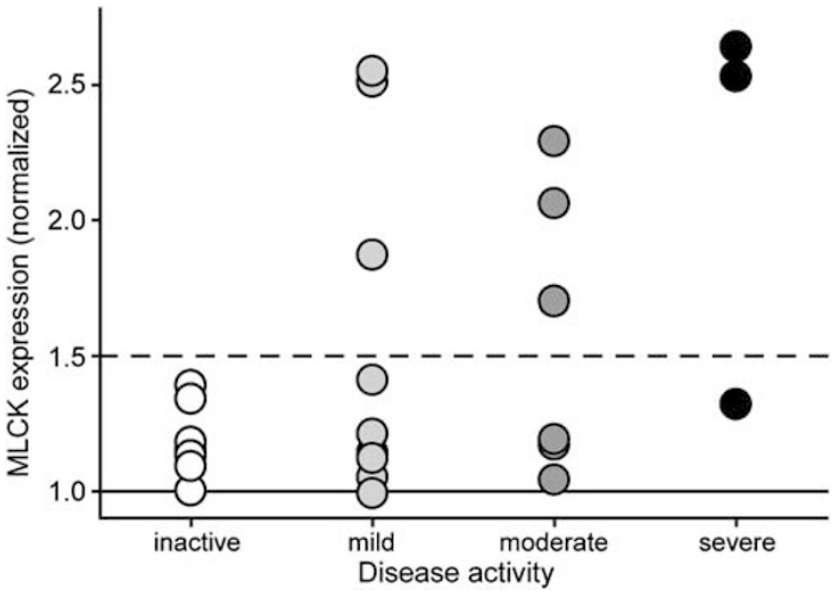

Figure 3 Ileal epithelial MLCK expression in CD correlates with disease activity. Specimens were grouped based on histological grading of disease activity (as in Figure 1). All samples with inactive disease (white circles) had normalized MLCK expression between 1.0 and 1.5. Thus, a reference line at a value of 1.5 is shown as the upper limit of expression for inactive disease (dashed line). For the samples with mild activity (light gray circles) MLCK expression exceeded 1.5 in $30 \%$. In the samples with moderate activity (dark gray circles) MLCK expression exceeded 1.5 in $43 \%$. In the samples with severe activity (black circles) MLCK expression exceeded 1.5 in $67 \%$.

these biopsies. Thus, although in vitro data suggests that the observed increases in MLCK expression may be secondary to transcriptional regulation, we were not able to test that hypothesis in these tissues. 
Ileal Epithelial MLCK Expression Correlates with Disease Activity within Individual CD Patients

Although analysis of the group data above suggests that MLCK expression correlates with disease activity, the data do not differentiate between systemic and local factors that relate activity to MLCK expression. For example, one might hypothesize that ileal epithelial MLCK expression is increased systemically in response to disease activation, much like serum acute phase reactants. Alternatively, one could equally well hypothesize that local factors regulate ileal epithelial MLCK expression. To discriminate between these possibilities we analyzed ileal epithelial MLCK expression in the six patients studied where multiple ileal specimens with varying disease activity were available. Two biopsies existed for five of these six patients and three existed for one patient, allowing analysis of 13 biopsies. MLCK expression increased with disease activity in four of these six patients (Figure 4). In the remaining two patients MLCK expression was similar in biopsies with more or less activity. Thus, these data suggest that ileal epithelial MLCK expression is regulated locally in parallel with disease activity.

\section{Ileal Epithelial MLCK1 Isoform Expression is Upregulated in Active CD}

We have recently described the limited expression of a specific MLCK isoform, MLCK1, in villous



Figure 4 Ileal epithelial MLCK expression correlates with disease activity in individual patients. Six of the CD patients studied had multiple sites sampled within the ileum that varied in disease activity. Each patient is represented by a unique symbol. In four of six patients normalized MLCK expression was significantly greater in areas with increased disease activity. In the remaining two patients (upright and inverted triangles) MLCK expression levels were similar in areas with varied disease activity. enterocytes. ${ }^{18}$ This isoform is localized specifically to the apical actomyosin ring where it regulates epithelial barrier function by modifying tight junction permeability. ${ }^{18}$ We therefore asked if this MLCK isoform was increased in ileal epithelium of CD patients. MLCK1 expression was analyzed in $18 \mathrm{CD}$ specimens and three controls. Quantitative analysis showed that, in those cells expressing MLCK1, expression increased modestly with histological activity (Figure 5a); normalized expression increased from $1.03 \pm 0.07$ in inactive and mildly active specimens $(n=10)$ to $1.24 \pm 0.11$ in moderate and severely active specimens $(n=8, P=0.04)$. These increases in MLCK1 expression correlated positively with increases in MLCK expression $(r=0.72)$, suggesting that MLCK1 is an important component of total MLCK expression and that factors regulating total MLCK expression may also regulate MLCK1 expression.

However, more striking than quantitative increases in MLCK1 expression were the qualitative changes in MLCK1 distribution associated with moderate and severe disease activity. MLCK1 expression was concentrated in cells at the villus tip and tightly restricted to the apical actomyosin ring in control tissue and tissue with inactive and mildly active disease (Figure 5b). Thus, MLCK1 was detected in less than $15 \%$ of enterocytes in ileal specimens with inactive and mildly active disease. In contrast, MLCK1 was readily detected along the surface of distorted villi in cases with moderate and severe disease activity, representing approximately $50 \%$ of enterocytes (Figure 5b). In these cases there also appeared to be some MLCK1 detected in the cytoplasm in areas distinct from the apical actomyosin ring. Thus, MLCK1 expression was increased in moderate and severely active CD, in terms of both the number of ileal enterocytes expressing MLCK1 and the magnitude of expression within individual enterocytes.

\section{Colonic Epithelial MLCK and MLCK1 Expression are Increased in Active UC and CD}

MLCK upregulation in small intestinal enterocytes of patients with active CD is consistent with a variety of experimental observations, including our recent report that TNF-induced MLCK-dependent epithelial barrier dysfunction occurs in murine small intestine, ${ }^{12}$ the observation that small intestinal barrier function is compromised in CD patients, ${ }^{4}$ and a report suggesting that anti-TNF therapy can restore barrier function in CD patients. ${ }^{8}$ In contrast, barrier function in the colon and in UC patients has been less well studied. Thus, we asked if increased MLCK and MLCK1 expression also occurred in colonic epithelium in the setting of active IBD. We examined 21 resection specimens from 11 patients; five with UC and six with CD. As described for ileal enterocytes, MLCK expression in 

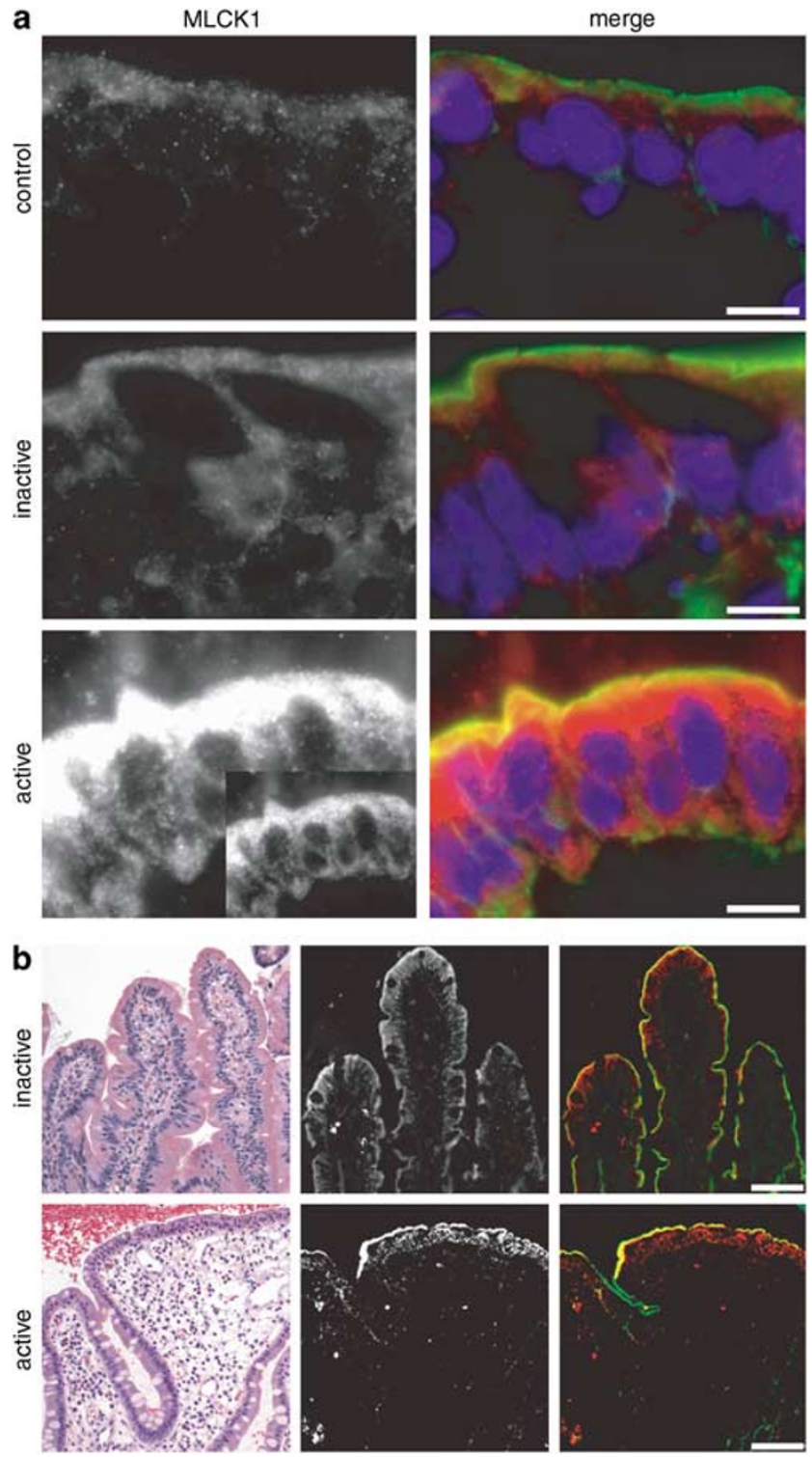

Figure 5 Ileal epithelial MLCK1 isoform expression is increased in CD. (a) In control tissue from a non-CD patient MLCK1 (red in the merged image) expression was limited, although detectable, at the apical actomyosin ring of villus enterocytes. F-actin (green) and nuclei (blue) are shown in the merged image for orientation. In CD tissue with inactive disease MLCK1 expression was also limited and concentrated at the apical actomyosin ring (exposures are matched throughout). In tissue with moderate activity MLCK1 expression was markedly increased. This resulted in saturation of the image when exposures were identical to those used for tissue without disease or with mild activity. The inset shows a $50 \%$ reduction of exposure for analysis of MLCK1 localization in tissue with severe disease. Bar $=10 \mu \mathrm{m}$. (b) Lower magnification images show that MLCK1 expression is restricted to villus enterocytes in inactive CD. Hematoxylin- and eosin-stained tissue from an adjacent section is shown for orientation. In contrast to inactive disease, MLCK1 is diffusely expressed along the surface in the presence of severe activity, with the result being a dramatic increase in the number of cells expressing MLCK1. Bar $=100 \mu \mathrm{m}$.

colonic surface epithelium was primarily localized to the apical actomyosin ring with limited amounts detected throughout the remainder of the cytoplasm
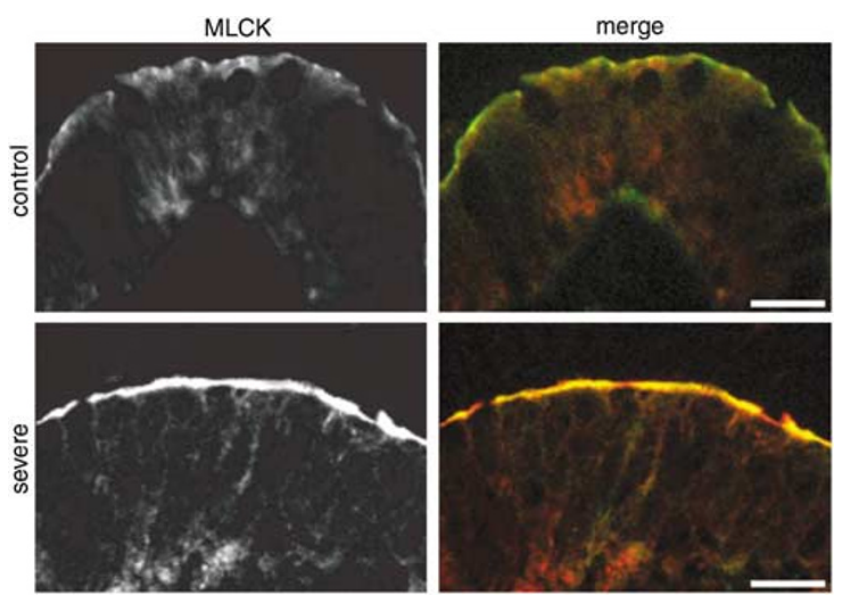

Figure 6 Colonic epithelial MLCK expression is increased in CD and UC. In control tissue from a UC with inactive disease MLCK expression (red in the merged image) was detectable at the apical actomyosin ring and within the cytoplasm of surface enterocytes. F-actin (green) is shown in the merged image for orientation. In UC tissue with active disease MLCK expression was increased at both subcellular locations. Results were similar in patients with colonic CD. Bar $=10 \mu \mathrm{m}$.

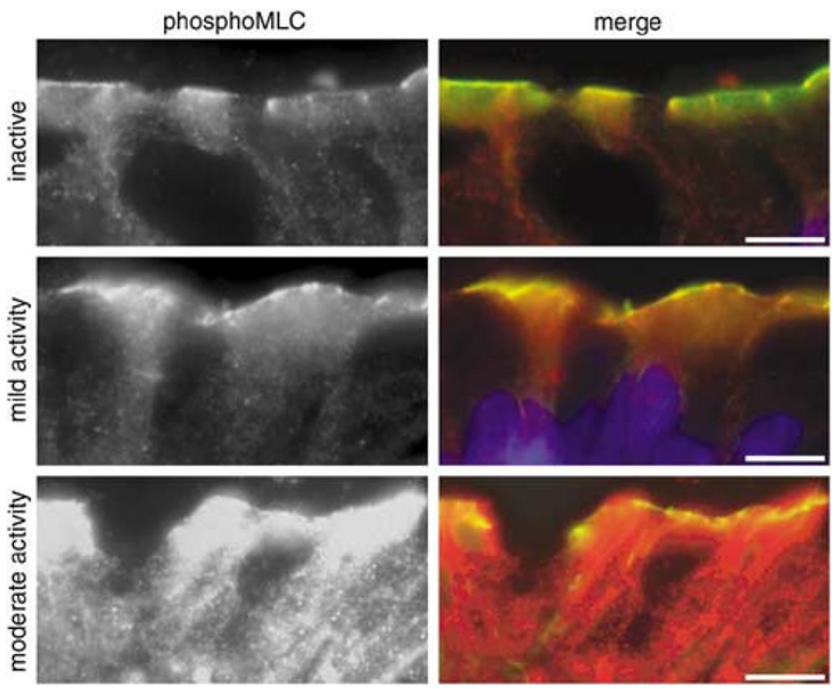

Figure 7 Colonic epithelial MLC phosphorylation is increased in CD and UC. In a biopsy from a UC patient with inactive disease the degree of MLC phosphorylation (red in the merged image) was similar to that seen in colonocytes in control (non-IBD). F-actin (green) and nuclei (blue) are shown in the merged image for orientation. Phosphorylated MLC (phosphoMLC) was primarily localized to the apical actomyosin ring at the site of the intercellular junction and was also present as a light diffuse staining within the apical cytoplasm. In a representative CD biopsy with mild activity and a UC biopsy with moderate activity the degree of MLC phosphorylation in both apical actomyosin ring and diffuse cytoplasmic pools increased progressively with increasing activity. Bar $=5 \mu \mathrm{m}$.

(Figure 6). In cases with inactive disease and mildly active disease, MLCK expression was not significantly different than in control cases $(1.02 \pm 0.11$, $n=9, P=0.2$ relative to controls). However, epithelial MLCK expression was markedly increased in 
cases with moderate and severe activity. In these cases normalized MLCK expression increased significantly to $1.46 \pm 0.11(n=12, P=0.005$ relative to cases with inactive disease and mild activity). When stratified by diagnosis there were no significant differences between MLCK expression levels in UC and CD patients. Similarly, there was no difference in MLCK expression when samples were stratified by site within the colon. Thus, like small intestinal epithelium, colonic epithelial MLCK expression is increased in IBD patients with moderate and severe disease activity.

Among the five UC patients studied, four had multiple specimens with differing activity (inactive/ mild vs moderate/severe) available for analysis. MLCK expression was increased in the more active specimens for three of these four patients, with no significant difference noted for the fourth patient. Thus, similar to ileum, colonic epithelial MLCK expression appears to be controlled by local factors.

\section{Increased MLCK Activity Accompanies Increases in MLC Expression}

The data above clearly demonstrate that intestinal epithelial MLCK protein expression is upregulated in active UC and CD. However, MLCK enzymatic activity is regulated by several mechanisms that are independent of protein expression. Thus, increased MLCK protein expression does not necessarily indicate increased kinase activity. To assess in vivo MLCK activity we measured phosphorylation of the endogenous MLCK substrate, MLC. While it might be correctly argued that phosphatase activity also contributes to overall levels of MLC phosphorylation, our recent in vitro and in vivo studies suggest that MLCK is the primary regulator of intestinal epithelial MLC phosphorylation. ${ }^{11,12}$ Unfortunately, our preliminary experiments showed that MLC phosphorylation decreased progressively with increasing time after resection, perhaps due to tissue anoxia, decreasing ATP levels, and unopposed phosphatase activity. To avoid this artifact we measured intestinal epithelial MLC phosphorylation in colon biopsies snap frozen within 1 min of resection. A total of 17 biopsies from 12 patients, seven with UC and five with CD, were studied.

Of the 17 biopsies studied, 10 had increased MLC phosphorylation, typically two- to three-fold greater than that seen in the control tissue (Figure 7). MLC phosphorylation was similar to controls in the remaining seven biopsies. When stratified into groups based on disease activity, increased MLC phosphorylation was present in seven of eight cases with active colitis but only in three of nine cases with inactive disease. Thus, MLC phosphorylation is increased in active colitis $(P=0.02)$. Consistent with this, one UC patient had several biopsies with differing disease activity. In this patient with three biopsies, two biopsies were inactive and one had moderate disease activity. One of the inactive biopsies as well as the biopsy with active disease demonstrated increased MLC phosphorylation, while MLC phosphorylation was similar to the controls in the third (inactive) biopsy. Thus, these group and individual data suggest that, like MLCK expression, MLCK activity is locally regulated and correlates with disease activity.

Based on in vitro ${ }^{16,17}$ and in vivo ${ }^{12}$ data linking intestinal epithelial MLC phosphorylation to TNF, we asked if there was any association between MLC phosphorylation and previous therapy using TNF antagonists. For example, the one case of active colitis without increased MLC phosphorylation might have been a patient treated with TNFneutralizing antibodies. However, the data show that this patient had not been treated with TNFneutralizing antibodies and no other associations between previous therapy and MLC phosphorylation status were apparent in our analyses of the patients whose biopsies were studied.

\section{Discussion}

While the presence of intestinal epithelial barrier dysfunction in CD and UC is generally accepted, neither the relevance to disease pathobiology nor the mechanisms of this barrier dysfunction are understood. This lack of mechanistic understanding has prevented definition of the role of barrier dysfunction in disease and limited exploration of barrier restoration as a therapeutic goal. Until recently, few clues have been available to explain the observed epithelial barrier defects. However, a variety of potential mechanisms have been proposed on the basis of in vitro and in vivo studies. These include epithelial apoptosis, mucosal ulceration, and altered tight junction protein expression.,12,22-27 While apoptosis and ulceration can certainly disrupt the mucosal barrier, abundant experimental evidence suggests that these alone cannot explain the barrier dysfunction observed. ${ }^{23}$

One pair of recent reports has shown that increased expression of the tight junction protein claudin-2 can contribute to the reduced barrier function seen in response to IL-13, both in vitro and in vivo. ${ }^{23,23}$ This may therefore explain some of the barrier dysfunction seen in UC, where IL-13 production by mucosal lymphocytes is markedly elevated..$^{23}$ However, another series of studies has clearly shown that TNF can induce apoptosisindependent barrier dysfunction in vitro and in vivo. ${ }^{11,12,16,17,25,26}$ While the mechanism of TNFinduced barrier dysfunction does not appear to involve increased claudin-2 expression, ${ }^{22}$ both in vitro ${ }^{11,16,17}$ and in vivo ${ }^{12}$ TNF-induced barrier dysfunction require MLCK activation. This is consistent with the documented role of MLCK in regulating epithelial tight junction permeability in 
response to physiological and pathophysiological stimuli in both in vitro and in vivo models. ${ }^{13,15,19,27}$

The mechanism by which TNF induces epithelial MLCK activation is not well understood, ${ }^{12}$ but studies in cultured epithelial monolayers suggest a role for TNF-dependent transcriptional activation of MLCK. ${ }^{16,17}$ As a result, both MLCK expression and activity are increased, as demonstrated by increased MLC phosphorylation. ${ }^{16,17}$ Inhibition of this increased MLCK activity restores barrier function in TNF-treated cultured epithelial monolayers, consistent with a central role for MLCK upregulation in TNF-induced barrier dysfunction.

To evaluate whether these in vitro observations are relevant to barrier dysfunction in human disease we studied patients with CD and UC, diseases in which barrier defects are well described ${ }^{7,28-31}$ and the therapeutic efficacy of TNF antagonism suggests that this cytokine plays an important pathogenic role. ${ }^{8,32-34}$ Our data show that, in both ileum and colon, MLCK expression is increased in a subset of patients with CD and UC. We also examined expression of the MLCK1 isoform, which is specifically associated with the tight junction in intestinal enterocytes. Like total MLCK, MLCK1 isoform expression was also increased in a subset of the patients studied. To determine if these increases in protein expression were accompanied by increases in MLCK enzymatic activity, we evaluated phosphorylation of the MLCK substrate MLC. Owing to the lability of phosphorylated MLC we found it impossible to study MLC phosphorylation in the resection specimens used for evaluation of MLCK expression. Thus, we used colon biopsy specimens snap frozen within 1 min of endoscopic resection. These proved suitable and, like MLCK protein expression, we found that MLC phosphorylation was increased in subset of patients with CD and UC.

To determine what separated the subset of patients with increased MLCK expression and activity from those without we evaluated clinical data, including diagnosis, biopsy site, previous and current therapy, previous surgery, smoking history, and duration of disease. None of these correlated, either positively or negatively, with increased MLCK expression or activity. However, when we assessed disease activity, as assessed histologically, we found that the magnitude of MLCK expression and presence of increased MLC phosphorylation correlated strongly with active inflammation. Analysis of patients with multiple samples confirmed an association between disease activity and MLCK expression and, strikingly, demonstrated that the degree of MLCK upregulation paralleled the degree of activity at each site. These data therefore suggest that MLCK upregulation may be directly related to disease activity. Moreover, the data suggest that the degree to which MLCK is upregulated can be controlled locally.

Despite the conclusion that MLCK expression and activity can be locally regulated, the data do not allow us to distinguish between cause and effect. For example, one might conclude from these data that MLCK is upregulated as a consequence of local active inflammation. This would certainly be consistent with the data presented and would also be supported by the observation that TNF can induce MLCK upregulation in cultured epithelia. Alternatively, one could hypothesize that the active inflammation is a consequence of MLCK upregulation. This would be plausible if one assumed that MLCK upregulation leads to barrier dysfunction, leakage of noxious and antigenic luminal contents into the lamina propria, and induction of an active inflammatory response. This model is also consistent with the data presented and would be supported by studies showing that barrier dysfunction is present in a subset of asymptomatic healthy relatives of CD patients as well as studies showing that barrier dysfunction predicts disease reactivation in CD patients in clinical remission. A third possibility that incorporates the first two might also be considered. We have recently proposed that barrier dysfunction and mucosal immune activation may be linked in a cycle. ${ }^{1}$ In this model, barrier dysfunction, whether primary or secondary, results in increased leakage of luminal contents and immune activation. This increased immune activation feeds back on the intestinal epithelium, as might occur due to cytokine-driven MLCK upregulation, to cause further loss of barrier function, further luminal leakage, and further immune activation. In this manner a self-amplifying cycle could be established, leading to disease in susceptible individuals. Discrimination between these and other possibilities will require further study. For example, analysis of biopsy specimens taken from patients at the time of diagnosis, prior to therapy, might shed light on the sequence of events leading to increases in intestinal epithelial MLCK expression and activity. However, in the modern era such studies are not possible for many reasons. First, only rare patients are biopsied on initial presentation. Thus, biopsies at time of diagnosis, while often prior to therapy, already represent chronic disease. Indeed, even if patients were biopsied at presentation, the available data suggest that histologically identifiable disease has already been present for some time. ${ }^{35}$ Second, the need for freshly frozen tissue demands that such a study be performed prospectively, with an assistant standing by to rapidly freeze tissues on all potential IBD diagnoses. Despite this, the recognition that MLCK expression and activity are increased in IBD represents an essential step forward in understanding the relationship between disease activity, MLCK upregulation, and disease pathogenesis.

In summary, the data presented demonstrate that MLCK expression and activity are upregulated in ileal and colonic epithelia involved by CD and UC. Moreover, the degree of upregulation correlates positively with the degree of active inflammation, suggesting an important relationship between these events. 


\section{Acknowledgements}

We are grateful to Drs Stephen Hanauer, David Rubin, Alessandro Fichera, Roger Hurst, and Fabrizio Michelassi for sharing their patients with us, Jonathan Bakst, Mary McCabe, Le Shen, and Edwina Witkowksi for their help with tissue acquisition, processing, and imaging, and the Pathology residents and endoscopy nurses of The University of Chicago Hospitals for their invaluable assistance. SAB was supported by a Howard Hughes Medical Institute student fellowship. DRC is a predoctoral fellow of the National Institutes of Health (The University of Chicago Medical Scientist Training Program T32 GM07281). This work was supported by the National Institutes of Health (R01DK61931 and RO1DK68271 to JRT), The University of Chicago Digestive Disease Center (P30DK42086), the Crohn's \& Colitis Foundation of America (to JRT), and the David and Reva Logan Center for Clinical Research in Gastroenterology at The University of Chicago.

\section{References}

1 Clayburgh DR, Shen L, Turner JR. A porous defense: the leaky epithelial barrier in intestinal disease. Lab Invest 2004;84:282-291.

2 Hollander D. Crohn's disease-a permeability disorder of the tight junction? Gut 1988;29:1621-1624.

3 Katz KD, Hollander D, Vadheim CM, et al. Intestinal permeability in patients with Crohn's disease and their healthy relatives. Gastroenterology 1989;97:927-931.

4 Ukabam SO, Clamp JR, Cooper BT. Abnormal small intestinal permeability to sugars in patients with Crohn's disease of the terminal ileum and colon. Digestion 1983;27:70-74.

5 Irvine EJ, Marshall JK. Increased intestinal permeability precedes the onset of Crohn's disease in a subject with familial risk. Gastroenterology 2000;119:17401744.

6 Wyatt J, Vogelsang H, Hubl W, et al. Intestinal permeability and the prediction of relapse in Crohn's disease. Lancet 1993;341:1437-1439.

7 Yacyshyn BR, Meddings JB. CD45RO expression on circulating CD19+ B cells in Crohn's disease correlates with intestinal permeability. Gastroenterology 1995; 108:132-137.

8 Suenaert P, Bulteel V, Lemmens L, et al. Anti-tumor necrosis factor treatment restores the gut barrier in Crohn's disease. Am J Gastroenterol 2002;97:20002004.

9 Hiribarren A, Heyman M, L'Helgouac'h A, et al. Effect of cytokines on the epithelial function of the human colon carcinoma cell line HT29 cl 19A. Gut 1993; 34:616-620.

10 Taylor CT, Dzus AL, Colgan SP. Autocrine regulation of epithelial permeability by hypoxia: role for polarized release of tumor necrosis factor alpha. Gastroenterology 1998;114:657-668.

11 Zolotarevsky Y, Hecht G, Koutsouris A, et al. A membrane-permeant peptide that inhibits MLC kinase restores barrier function in in vitro models of intestinal disease. Gastroenterology 2002;123:163-172.
12 Clayburgh DR, Barrett TA, Tang Y, et al. Epithelial myosin light chain kinase-dependent barrier dysfunction mediates $\mathrm{T}$ cell activation-induced diarrhea in vivo. J Clin Invest 2005;115:2702-2715.

13 Turner JR, Rill BK, Carlson SL, et al. Physiological regulation of epithelial tight junctions is associated with myosin light-chain phosphorylation. Am J Physiol 1997;273:C1378-C1385.

14 Scott KG, Meddings JB, Kirk DR, et al. Intestinal infection with Giardia spp. reduces epithelial barrier function in a myosin light chain kinasedependent fashion. Gastroenterology 2002;123:11791190.

15 Yuhan R, Koutsouris A, Savkovic SD, et al. Enteropathogenic Escherichia coli-induced myosin light chain phosphorylation alters intestinal epithelial permeability. Gastroenterology 1997;113:1873-1882.

16 Wang F, Graham WV, Wang Y, et al. Interferon-gamma and tumor necrosis factor-alpha synergize to induce intestinal epithelial barrier dysfunction by up-regulating myosin light chain kinase expression. Am J Pathol 2005;166:409-419.

17 Ma TY, Boivin MA, Ye D, et al. Mechanism of TNFalpha modulation of Caco-2 intestinal epithelial tight junction barrier: role of myosin light-chain kinase protein expression. Am J Physiol Gastrointest Liver Physiol 2005;288:G422-G430.

18 Clayburgh DR, Rosen S, Witkowski ED, et al. A differentiation-dependent splice variant of myosin light chain kinase, MLCK1, regulates epithelial tight junction permeability. J Biol Chem 2004;279: 55506-55513.

19 Berglund JJ, Riegler M, Zolotarevsky Y, et al. Regulation of human jejunal transmucosal resistance and MLC phosphorylation by $\mathrm{Na}^{+}$-glucose cotransport. Am J Physiol Gastrointest Liver Physiol 2001;281: G1487-G1493.

20 Graham WV, Wang F, Wang Y, et al. AP1-dependent transcriptional activation of myosin light chain kinase mediates IFNgamma- and TNFalpha-induced barrier dysfunction. FASEB J 2005;19, Abstract.

21 Micke P, Ohshima M, Tahmasebpoor S, et al. Biobanking of fresh frozen tissue: RNA is stable in non-fixed surgical specimens. Lab Invest 2006;86 (in press).

22 Prasad S, Mingrino R, Kaukinen K, et al. Inflammatory processes have differential effects on claudins 2,3 and 4 in colonic epithelial cells. Lab Invest 2005;85: 1139-1162.

23 Heller F, Florian P, Bojarski C, et al. Interleukin-13 is the key effector Th2 cytokine in ulcerative colitis that affects epithelial tight junctions, apoptosis, and cell restitution. Gastroenterology 2005;129:550-564.

24 Gitter AH, Bendfeldt K, Schulzke JD, et al. Leaks in the epithelial barrier caused by spontaneous and TNFalpha-induced single-cell apoptosis. FASEB J 2000;14: 1749-1753.

25 Musch MW, Clarke LL, Mamah D, et al. $\mathrm{T}$ cell activation causes diarrhea by increasing intestinal permeability and inhibiting epithelial $\mathrm{Na}+\mathrm{K}+\mathrm{AT}-$ Pase. J Clin Invest 2002;110:1739-1747.

26 Bruewer M, Luegering A, Kucharzik T, et al. Proinflammatory cytokines disrupt epithelial barrier function by apoptosis-independent mechanisms. J Immunol 2003;171:6164-6172.

27 Ma TY, Nguyen D, Bui V, et al. Ethanol modulation of intestinal epithelial tight junction barrier. Am J Physiol 1999;276:G965-G974. 
28 Hollander D. Permeability in Crohn's disease: altered barrier functions in healthy relatives? Gastroenterology 1993;104:1848-1851.

29 Meddings JB, Sutherland LR, May GR. Intestinal permeability in patients with Crohn's disease. Gut 1994;35:1675-1676.

30 Jenkins RT, Ramage JK, Jones DB, et al. Small bowel and colonic permeability to 51Cr-EDTA in patients with active inflammatory bowel disease. Clin Invest Med 1988;11:151-155.

31 Gitter AH, Wullstein F, Fromm M, et al. Epithelial barrier defects in ulcerative colitis: characterization and quantification by electrophysiological imaging. Gastroenterology 2001;121:1320-1328.

32 Jarnerot G, Hertervig E, Friis-Liby I, et al. Infliximab as rescue therapy in severe to moderately severe ulcera- tive colitis: a randomized, placebo-controlled study. Gastroenterology 2005;128:1805-1811.

33 Baert FJ, D'Haens GR, Peeters M, et al. Tumor necrosis factor alpha antibody (infliximab) therapy profoundly down-regulates the inflammation in Crohn's ileocolitis. Gastroenterology 1999;116: 22-28.

34 D’Haens G, Van Deventer S, Van Hogezand R, et al. Endoscopic and histological healing with infliximab anti-tumor necrosis factor antibodies in Crohn's disease: A European multicenter trial. Gastroenterology 1999;116:1029-1034.

35 Kumar NB, Nostrant TT, Appelman HD. The histopathologic spectrum of acute self-limited colitis (acute infectious-type colitis). Am J Surg Pathol 1982;6: 523-529. 